\title{
3D-printed titanium implant-coated polydopamine for repairing femoral condyle defects in rabbits
}

\author{
Weiyang Zhong ${ }^{1}$, Jianxiao $\mathrm{Li}^{1}$, Chenbo Hu${ }^{1}$, Zhengxue Quan ${ }^{1}$, Dianming Jiang ${ }^{2 *}$, Guangbin Huang ${ }^{3}$ and \\ Zhigang Wang ${ }^{4}$
}

\begin{abstract}
Background: Large segmental bone defects are still one of the challenges for orthopaedic surgeons. Although 3Dprinted porous titanium is a potential bone substitute material because of its porous structure simulating natural bone, the titanium surface has low bioactivity, integrates with bone tissue through the simple mechanical interlock. The study aims to investigate the capability and osteogenesis of 3D-printed porous titanium (3D PPT)-coated polydopamine (PDA) for repairing bone defects.

Methods: Fifteen 6-month New Zealand white rabbits were implanted with PDA-3D PPT to repair $6 \mathrm{~mm} \times 10 \mathrm{~mm}$ defects on the femoral condyle compared with the group of 3D PPT and comparing with the blank group. After 6 weeks and 12 weeks, micro-CT and histological examination were performed to observe bone growth.

Results: All the PDA-3D PPT group, the 3D PPT group and the blank group recovered in good condition. The images showed that the boundaries between the implant area and the surrounding area were obscure in the three groups. The results of micro-CT demonstrated that at 6 weeks and 12 weeks, the bone volume (BV) values of PDA3D PPT implants group were significantly higher than those of the 3D PPT implants group and blank group $(P<$ 0.05), the BV/tissue volume (TV) and the trabecular number (Tb.N) of PDA-3D PPT implants were significantly higher than those of the 3D PPT group and blank group $(P<0.05)$. The results of un-decalcified bone slicing showed that ore new bone appeared to form around the PDA-3D PPT than that of 3D PPT and blank group. The bone-implant contact $(\mathrm{BIC})$ of PDA-3D PPT was better $(P<0.05)$ than that of 3D PPT group.
\end{abstract}

Conclusion: PDA-3D PPT could improve the bioactivity and promote the growth and healing of bone tissue and can be a promising repairing material.

Keywords: 3D-printed porous titanium, Polydopamine, Bone defect

\section{Background}

Large segmental bone defects caused by high-energy trauma, infection, bone tumour or congenital malformation are still one of the challenges for orthopaedic surgeons. At present, the most common management strategies involve autologous bone grafts or allogeneic grafts, which have advantages and disadvantages. Autologous bone grafting requires additional surgery and more trauma, which often have complications, such as nerve

\footnotetext{
* Correspondence: jdm571026@vip.163.com

${ }^{2}$ Department of Orthopedic Surgery, The Third Affiliated Hospital of

Chongqing Medical University, Chongqing 400042, China

Full list of author information is available at the end of the article
}

damage, fractures and infections. Allogeneic bone grafts have the risks of immune diseases and of slowing bone remodelling [1-3]. 3D-printed porous titanium (3D PPT) is a potential bone substitute material because of its porous structure simulating natural bone, which is beneficial to the growth of new bone tissue and provides a new way to treat bone defects [4-8].

However, the titanium surface has low bioactivity, forms a simple mechanical interlock with the bone tissue and cannot integrate with the bone. Dopamine, which contains catecholamine functional groups and lysine end

(c) The Author(s). 2020 Open Access This article is distributed under the terms of the Creative Commons Attribution 4.0 International License (http://creativecommons.org/licenses/by/4.0/), which permits unrestricted use, distribution, and 


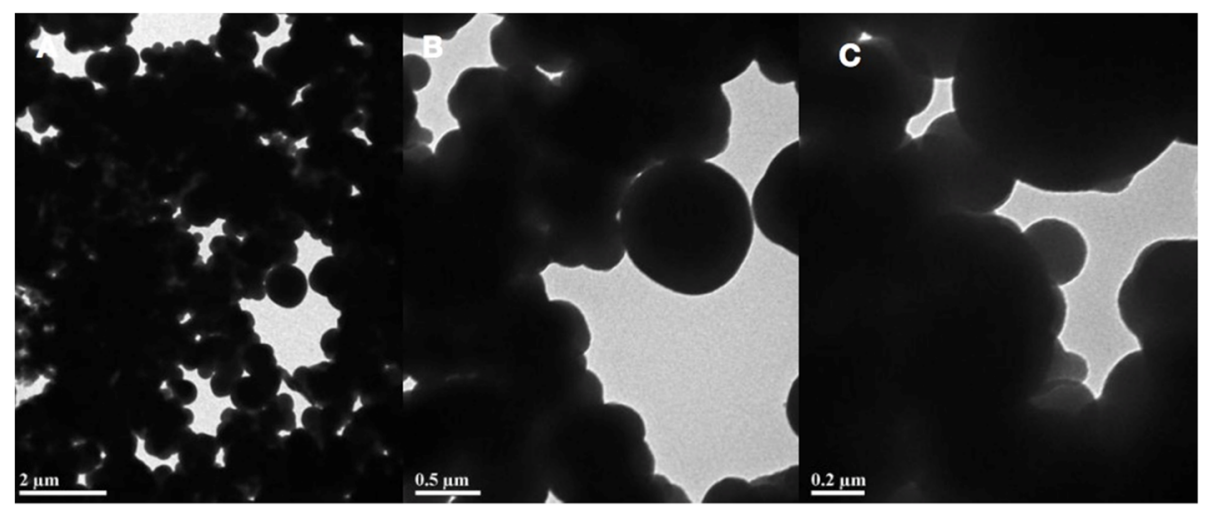

Fig. 1 SEM images of PDA. $\mathbf{a} \times 2 \mu \mathrm{m} ; \mathbf{b} \times 0.5 \mu \mathrm{m} ; \mathbf{c} \times 0.2 \mu \mathrm{m}$

amino groups, can form very strong adhesion dopamine (polydopamine, PDA) films on the surface of glass, metal, ceramics, organic matter and other materials under alkaline conditions. PDA can fix biomolecules on the surface of a modified material $[9,10]$.

We used a laser three-dimensional forming technology to process titanium powder to prepare 3D PPT coated by PDA, acquiring a good hydrophilic surface to use biochemical surface modification methods to modify the bone-implant surface with a high specific surface area and increasing the mechanical locking of the implant and the bone.

The capacity of the modified 3D PPT to repair femur defects was investigated in vivo, which could provide the experimental basis for clinical application.

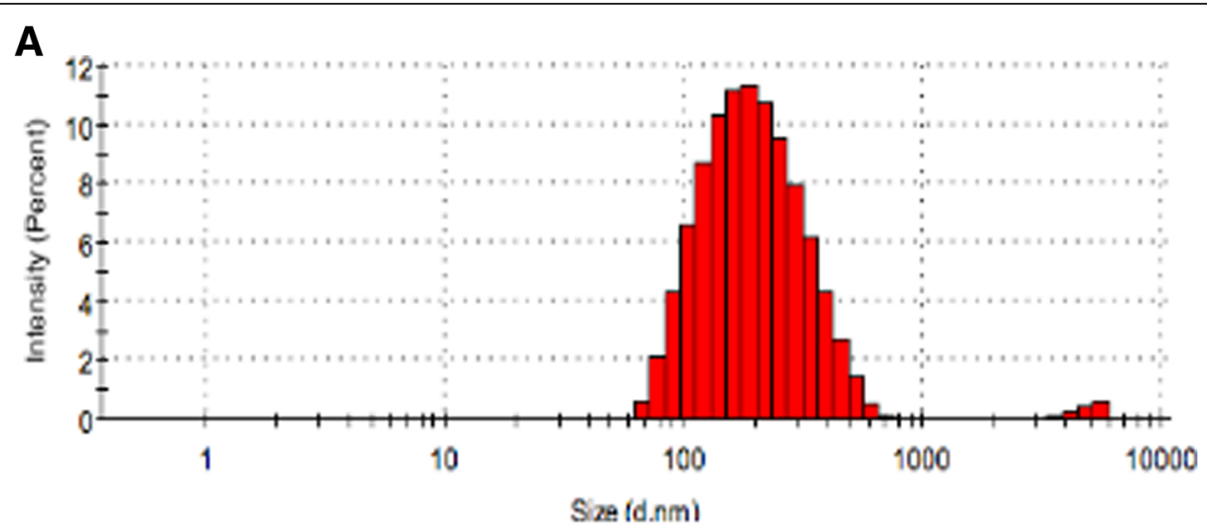

B

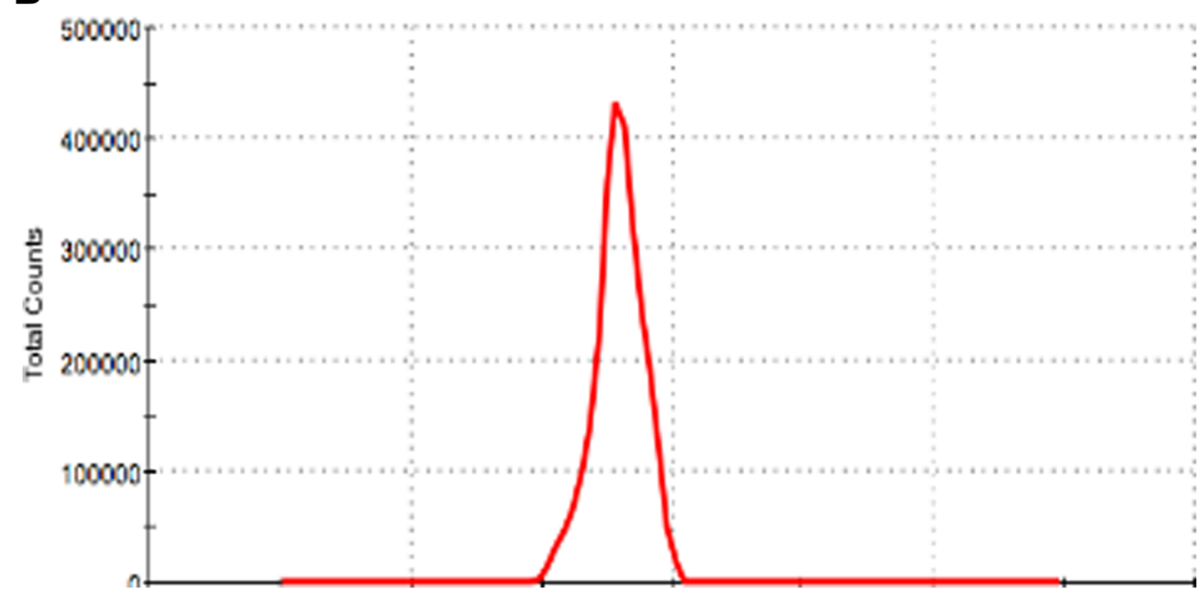

Fig. 2 Particle size and zeta potential of PDA. a Particle size; b zeta potential 


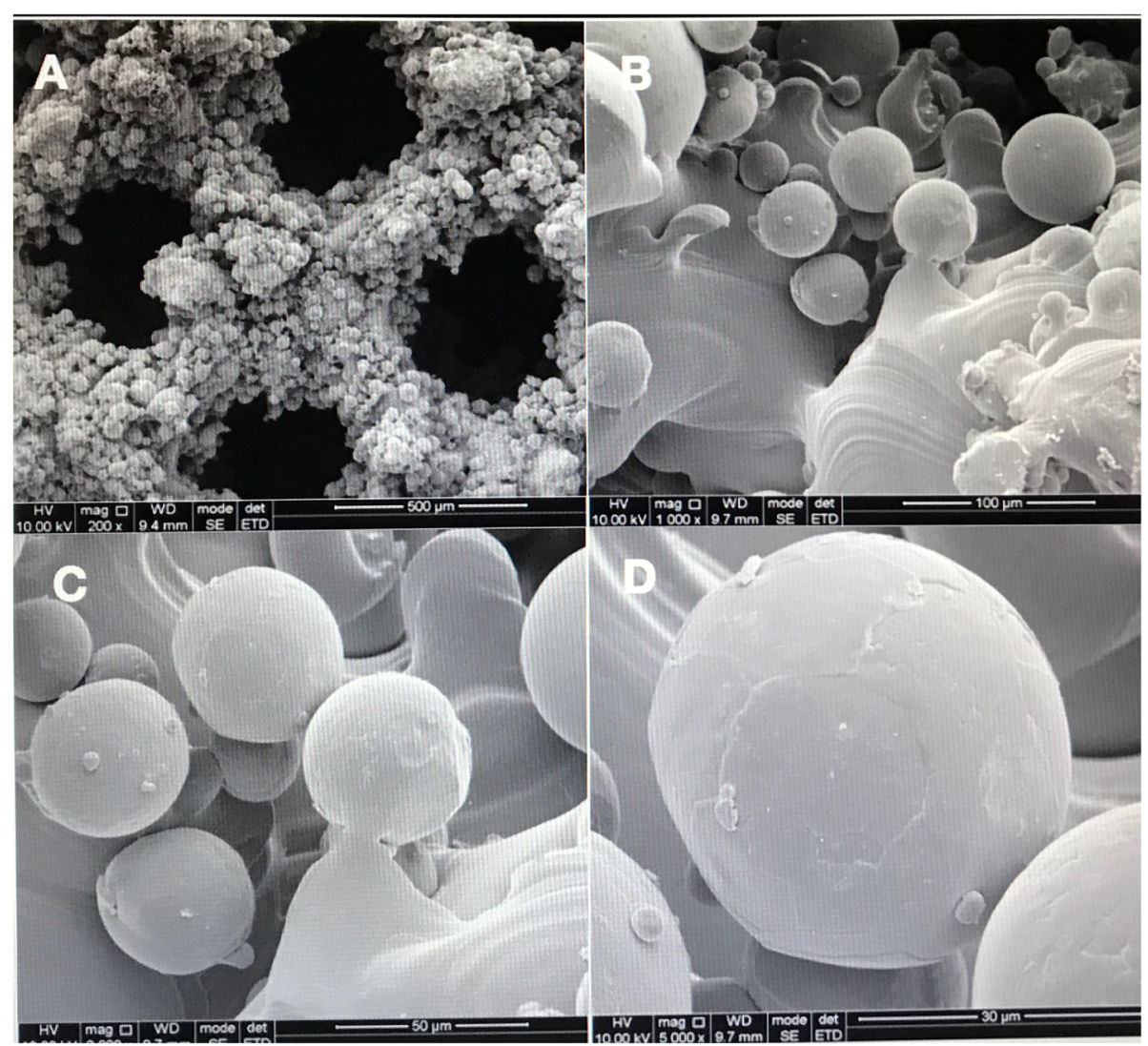

Fig. 3 SEM images of PDA-3D PPT. $\mathbf{a} \times 200 \mu \mathrm{m} ; \mathbf{b} \times 100 \mu \mathrm{m} ; \mathbf{c} \times 50 \mu \mathrm{m} ; \mathbf{d} \times 30 \mu \mathrm{m}$

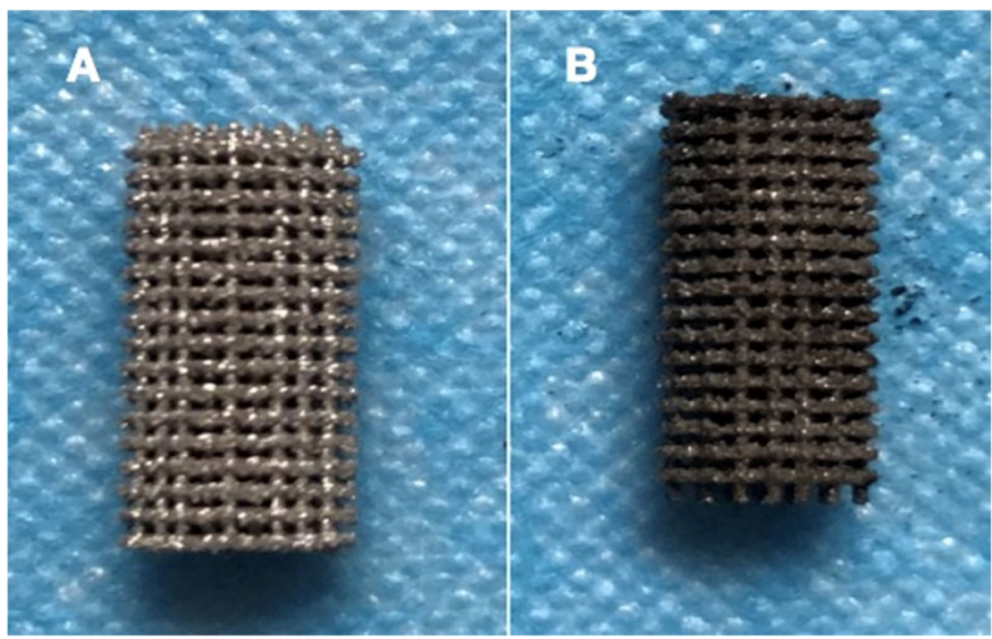

Fig. 4 Images of 3D PPT and PDA-3D PPT. a 3D PPT; b PDA-3D PPT 


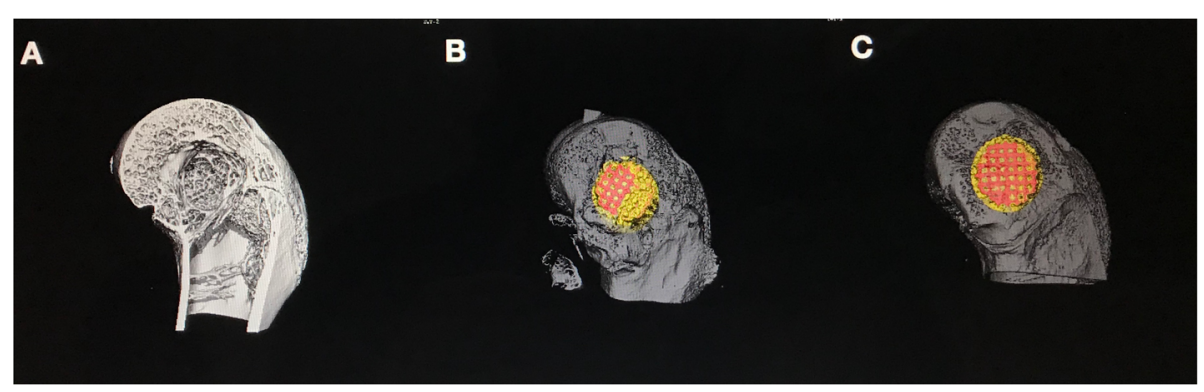

Fig. 5 Micro-CT 3D reconstruction images on the femoral defect. a Blank; b 3D PPT; c PDA-3D PPT

\section{Material and methods}

\section{Preparation of PDA-coated 3D PPT}

The preparation of the PDA coating was performed according to the described method [10,11] (Figs. 1 and 2). Titanium alloy (Ti6AL4V) implants prepared using selective laser melting and containing a three-dimensional implant architecture were designed as a cylindrical type (6.0 $\mathrm{mm}$ diameter $\times 10 \mathrm{~mm}$ long) and used for implantation.

\section{Material surface characterization}

The surface morphology of 3D PPT implant-coated polydopamine and 3D PPT implants was observed using scanning electron microscopy (SEM; JSM-7500; JEOL, Tokyo, Japan) to confirm the presence of the PDA coating on the surface of the 3D PPT (Figs. 3 and 4).

\section{Experimental animals and surgical procedure}

All animal experiment protocols were conducted according to the procedures approved by the Animal Care and Experiment Committee of The First Affiliated Hospital of Chongqing Medical University and were approved by the animal ethics committee of The First Affiliated Hospital of Chongqing Medical University (no: 20187801). Fifteen adult, male New Zealand white rabbits weighing $2.0-3.0 \mathrm{~kg}$ were applied in our study and were randomly divided into three groups $(\boldsymbol{n}=5$ each). The one-side femur of each rabbit was used as the surgical site. General anaesthesia was induced by intravenous injection
$(1.0 \mathrm{~mL} / \mathrm{kg})$ with a $3 \%$ pentobarbital sodium solution (Sigma-Aldrich Co.). After anaesthetisation, the surgical area was shaved and disinfected with iodine. After a longitudinal $3.5 \mathrm{~cm}$ lateral incision was made, the patella was dislocated medially, and the femoral groove was exposed. With the knees flexed, the bone defects $(6 \mathrm{~mm}$ width $\times 10 \mathrm{~mm}$ depth) were manually created in the femoral groove with a bone driller ( $4 \mathrm{~mm}$ width $\times 2 \mathrm{~mm}$ thickness). Then, the 3D PPT and PDA-3D PPT implants were installed in the defects and the blank group was without any implant. The incisions were sutured in layers, and the lateral ligaments were sutured tightly to avoid patellar dislocation. Penicillin sodium (Southwest Pharmaceutical Co., Ltd., Chongqing, PRC; 200,000 IU/ $\mathrm{kg} /$ day, intramuscular injection) was administered for 3 days postoperatively. The rabbits were kept in separate cages and were allowed full activity after surgery.

\section{Micro-CT measurements and histological evaluations}

Micro-CT scanning and histological staining were applied to observe bone formation around the implants at 6 and 12 weeks postoperatively.

\section{Statistical analysis}

Statistical analysis was performed using the Statistic Analysis System (SAS Institute Inc., Cary, NC, USA). Quantitative variables were described as the mean \pm SD. Student's $\boldsymbol{t}$ test was used for the statistical analysis of differences in mean values, and the chi-squared test was

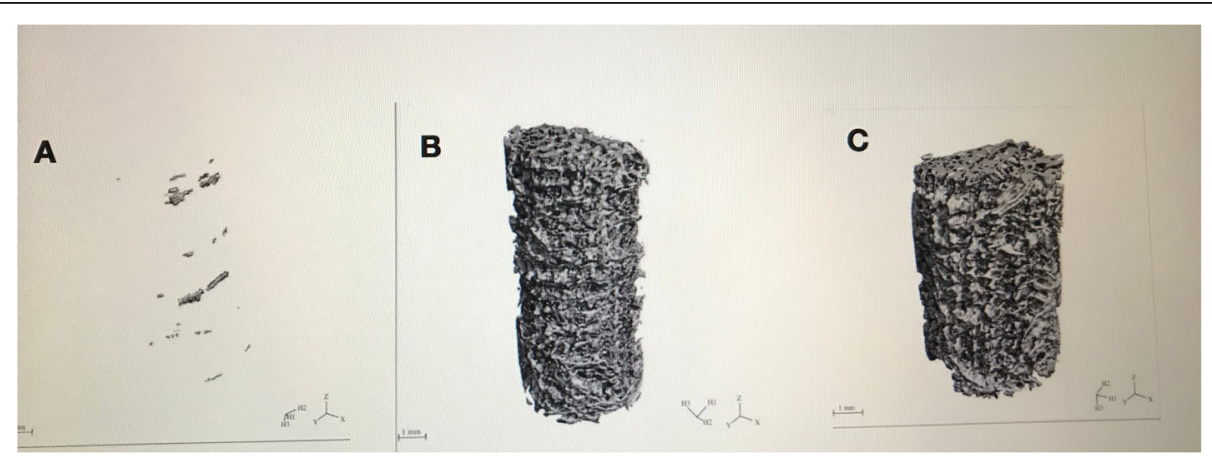

Fig. 6 Micro-CT 3D reconstruction images of 3D PPT and PDA-3D PPT of peri-implant bone with $1 \mathrm{~mm}$. a Blank; b 3D PPT; c PDA-3D PPT 


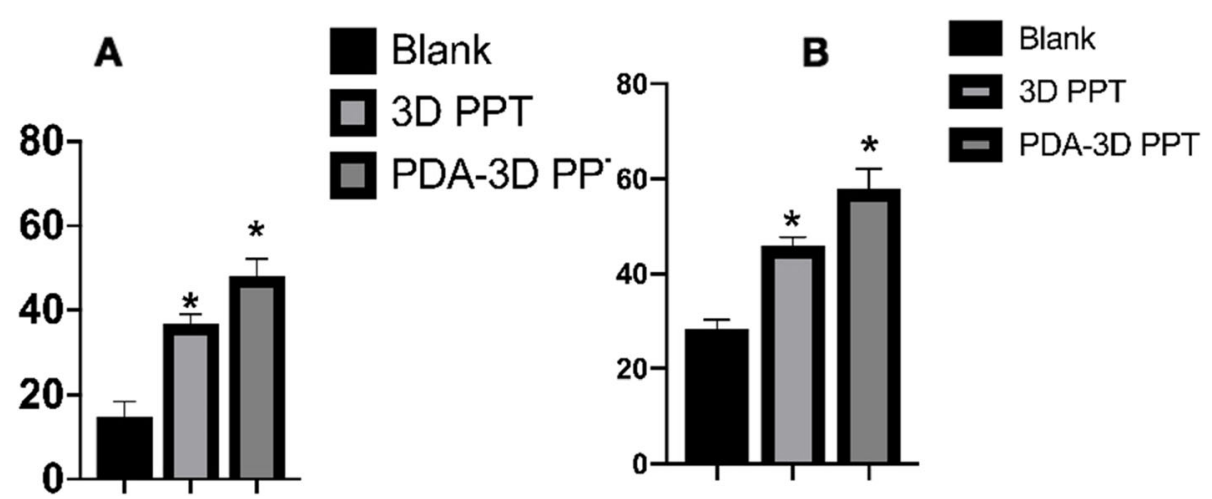

Fig. 7 BV shows significant differences in both groups. a 6 weeks; b 12 weeks

used for categorical data. Differences with a $\boldsymbol{P}$ value $<$ 0.05 were considered.

\section{Results}

After the implantation of the material, the general condition of the rabbits was good. The incisions had different degrees of swelling, but there were no infections. The rabbits were sacrificed 6 weeks and 12 weeks after the operation, and the specimens were removed. The new bone tissues were filled with the experimental bones.

Micro-CT revealed fibrous tissue growth in the bone defect area 6 weeks after surgery. Twelve weeks after surgery, the three groups of specimens showed different degrees of periosteal reaction in the bone defect area and the implant material, and a small amount of callus formation was observed. At 12 weeks postoperatively, the specimens were taken for micro-CT examination. The formation of calli was observed in all groups. The density of PDA-3D PPT implanted in the experimental group was higher than that in the 3D PPT group and blank group of the surrounding host bone, and more bone callus was formed. The boundary between the bone and its surrounding was blurred, and the porous area was filled.

The micro-CT 3D reconstruction images showed that more new bone appeared to form within the VOI around the PDA-3D PPT implants than that detected in other groups (Figs. 5 and 6). At 6 weeks and 12 weeks, the bone volume (BV) values of PDA-3D PPT implant group were significantly higher than those of the 3D PPT implants group and blank group $(P<0.05)$ (Fig. 7). To quantitatively analyse the formation of new bone, the BV/tissue volume(TV) and the trabecular number (Tb.N) of each group were determined by micro-CT analysis. As shown, the BV/TV values and Tb.N of PDA-3D PPT implants were significantly higher than those of the 3D PPT group and blank group $(P<0.05)$ (Figs. 8 and 9).

At 12 weeks after surgery, the PDA-3D PPT was tightly implanted with the surrounding bone tissue and was closely combined, and there was no obvious inflammatory cell infiltration or interfiber tissue. There was no obvious bone resorption or osteolysis in the implant material area. More new bone appeared to form around the PDA-3D PPT than that of 3D PPT and blank group (Fig. 10). The bone-implant contact (BIC) of PDA-3D PPT was better $(P$ $<0.05$ ) than that of the 3D PPT group (Fig. 11).

\section{Discussion}

Bone defects caused by trauma, infection, and tumour resection are common and seriously endanger bone

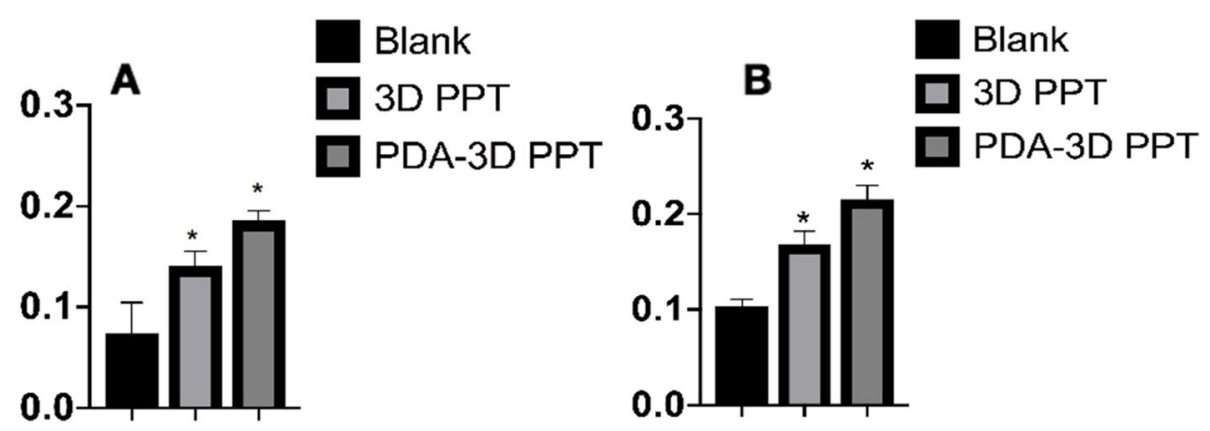

Fig. 8 The BV density values (BV/TV\%) are significantly different. a 6 weeks; b 12 weeks 

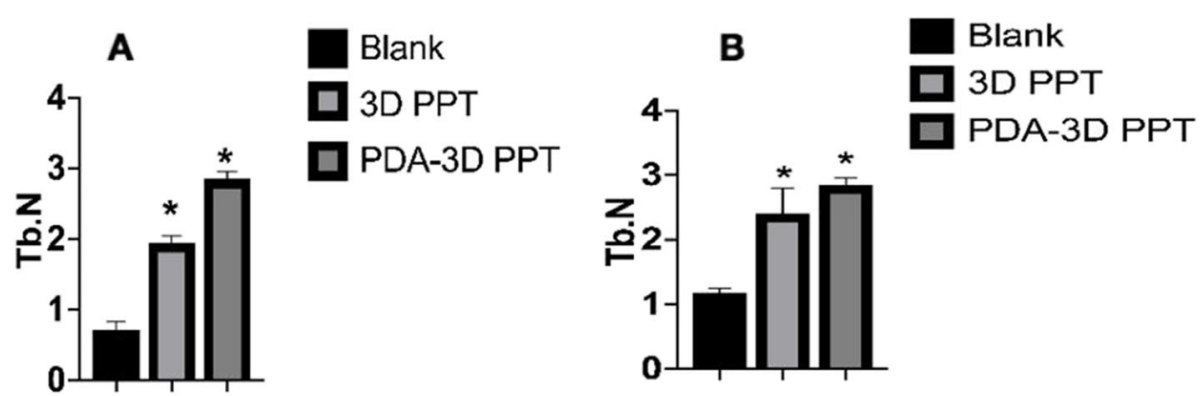

Fig. 9 The Tb.N are significantly different. a 6 weeks; b 12 weeks

healing [1-4]. Currently, porous titanium implants based on $3 \mathrm{D}$ printing have become a bone substitute because of their porous property and the open porous structure facilitates the transport of blood and nutrients in the implant, promoting tissue regeneration and reconstruction and speeds up the repair process [5-10]. However, the titanium surface has low bioactivity, forms a simple mechanical interlock with the bone tissue, and does not induce bone integration. The ideal goal of implant surface modification could achieve strong osseointegration and improve long-term stability and the osseointegration depends on direct interaction between the bone tissue and biomaterial surface. The 3D PPT coating is one of the most common methods for surface modification of biomaterials and can improve the process of osseointegration [11-16].

The PDA film was performed by dopamine can produce a polymerized layer on almost any shape and material type by oxidative self-polymerization under alkaline conditions. As shown in Figs. 1, 2, 3, 4, the mean diameter of PDA particle was $100-300 \mathrm{~nm}$ and the SEM images demonstrated significant numbers of spherical particles formed on the surface of the 3D PPT which increased greatly the surface area. The previous studies showed that PDA preparation on substrates can provide many hydroxyl groups $(\mathrm{OH}-)$, attracting calcium ions
$(\mathrm{Ca} 2+)$ and subsequently bond with phosphate ions (PO3-)which could improve the bioactivity of 3D PPT, forming a stronger interlock with the bone tissue [17-20].

Direct bone attachment and integration on the implant interface plays a key role in the implant performance. The effects of PDA coatings on osseointegration were further studied in vivo. In our study, micro-CT and histological examinations were conducted to detect new bone formation at the bone-implant interface. We observed that newly formed bone was tightly bound in blank group. Furthermore, the more newly formed bone could be observed at the bone-implant interface of PDA-3D PPT compared with 3D PPT. Quantitative analysis of micro-CT further confirmed that more new bone was formed around PDA-3D PPT than the other two groups. Histological examination and BIC resulted in the same conclusion. The results showed that PDA-3D PPT had a more positive effect on osseointegration in vivo than the 3D PPT.

\section{Conclusion}

In our study, a PDA coating was successfully prepared on the surface of a 3D PPT material. In vivo studies, micro-CT analysis and histological findings revealed that the PDA coating significantly improved the osteogenic

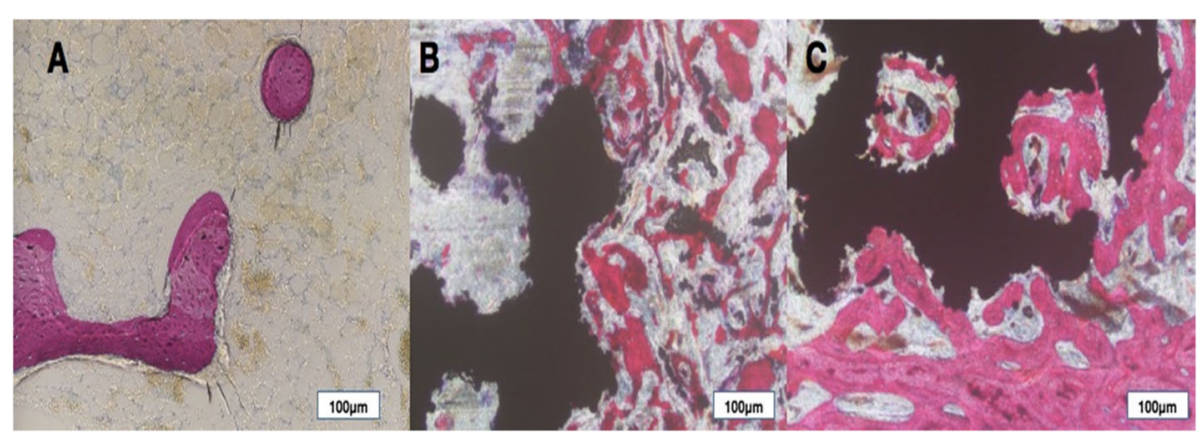

Fig. 10 Histological analysis (H\&E staining) of femoral condyle defects at 12 weeks after surgery. a Blank; b 3D PPT; c PDA-3D PPT 


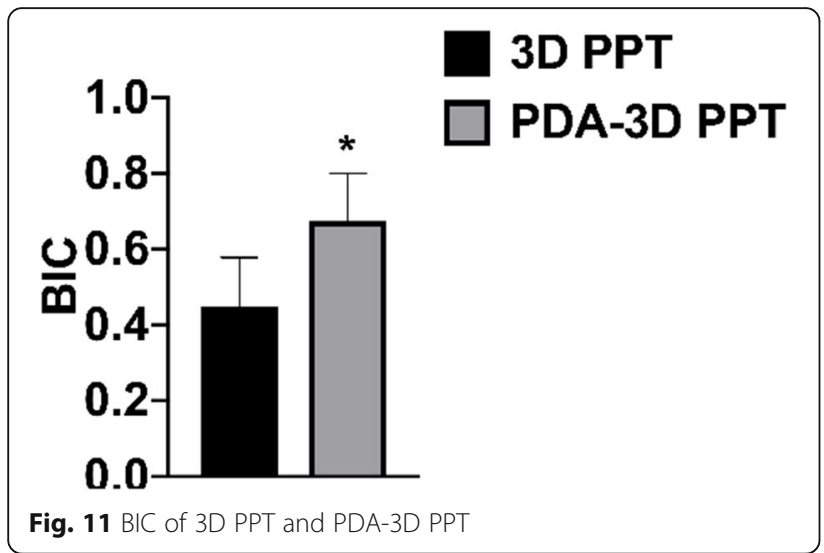

induction of PDA-3D PPT implants. In conclusion, PDA-3D PPT improved the osteogenesis-inducing ability of the biomaterials and provided the potential for the surfacemodification of implanted materials.

\section{Abbreviations}

3D PPT: 3D-printed porous titaniumBICBone-implant contactBVBone volume; PDA: Polydopamine; Tb.N: Trabecular number; TV: Tissue volume

\section{Acknowledgements}

WYZ acknowledges Haitao Peng for technical help in the experiment.

\section{Authors' contributions}

WYZ and DMJ designed the study. WYZ, JXL, CBH, ZXQ, GBH, and ZGW implemented the experiment. WYZ wrote the manuscript. All authors read and approved the final manuscript.

\section{Funding}

The study was supported by the medical senior talents programme of Chongqing(2016HBRC007)

\section{Availability of data and materials}

The datasets used and/or analyzed during the current study are available from the corresponding author on reasonable request.

\section{Ethics approval and consent to participate}

The Institutional Review Board of the First Affiliated Hospital of Chongqing Medical University approved this study.

\section{Consent for publication}

This paper is approved by all authors for publication.

\section{Competing interests}

The authors declare that they have no competing interests.

\section{Author details}

${ }^{1}$ Department of Orthopedic Surgery, The First Affiliated Hospital of Chongqing Medical University, Chongqing 400016, China. ${ }^{2}$ Department of Orthopedic Surgery, The Third Affiliated Hospital of Chongqing Medical University, Chongqing 400042, China. ${ }^{3}$ Department of Trauma Surgery, Emergency Medical Center of Chongqing, The Affiliated Central Hospital of Chongqing University, Chongqing 400014, China. ${ }^{4}$ Institute of Ultrasound Imaging, The Second Affiliated Hospital of Chongqing Medical University, Chongqing 400010, China.
Received: 8 October 2019 Accepted: 13 February 2020

Published online: 11 March 2020

\section{References}

1. Dou H, Wang $G$, Xing N, et al. Repair of large segmental bone defects with fascial flap-wrapped allogeneic bone. J Orthop Surg Res. 2016;11(1):162

2. Li JJ, Roohani-Esfahani SI, Dunstan CR, et al. Efficacy of novel synthetic bone substitutes in the reconstruction of large segmental bone defects in sheep tibiae. Biomed Mater. 2016:11(1):015016.

3. Wu D, Wang Z, Wang J, et al. Development of a micro-tissue-mediated injectable bone tissue engineering strategy for large segmental bone defect treatment. Stem Cell Res Ther. 2018;9(1):331.

4. Puleo DA, Nanci A. Understanding and controlling the bone-implant interface. Biomaterials. 1999;20(23-24):2311-21.

5. Bertol LS, Schabbach R, Loureiro Dos Santos LA. Different post-processing conditions for 3D bioprinted a-tricalcium phosphate scaffolds. J Mater Sci Mater Med. 2017:28:168.

6. Walsh WR,Pelletier MH,Wang T et al. Does implantation site influence bone ingrowth into 3D printed porous implants? Spine J. 2019 Jun 27;pii:S15299430(19)30837-X. [Epub ahead of print].

7. Tsai $\mathrm{CH}$, Hung $\mathrm{CH}$, Kuo $\mathrm{CN}$, et al. Improved bioactivity of $3 \mathrm{~d}$ printed porous titanium alloy scaffold with chitosan/magnesium-calcium silicate composite for orthopaedic applications. Materials (Basel). 2019;12(2): ma12020203.

8. Ma L, Wang X, Zhao N, et al. Integrating 3D printing and biomimetic mineralization for personalized enhanced osteogenesis, angiogenesis, and osteointegration. ACS Appl Mater Interfaces. 2018; [Epub ahead of print].

9. Bruining MJ, Pijpers AP, Kingshott P, Koole LH. Studies on new polymeric biomaterials with tunable hydrophilicity, and their possible utility in corneal repair surgery. Biomaterials. 2002;23(4):1213-9.

10. Al Qahtani WM, Schille C, Spintzyk S, et al. Effect of surface modification of zirconia on cell adhesion, metabolic activity and proliferation of human osteoblasts. Biomed Tech. 2017:62(1):75-87.

11. Van De Walle E, Van Nieuwenhove I, Vanderleyden E, et al. Polydopaminegelatin as universal cell-interactive coating for methacrylate-based medical device packaging materials: when surface chemistry overrules substrate bulk properties. Biomacromolecules. 2016:17(1):56-68.

12. Györgyey Á, Ungvári K, Kecskeméti G, et al. Attachment and proliferation of human osteoblast-like cells (MG-63) on laser-ablated titanium implant material. Mater Sci Eng C Mater Biol Appl. 2013;33(7):4251-9.

13. Lee $H$, Dellatore SM, Miller WM, Messersmith PB. Mussel-inspired surface chemistry for multifunctional coatings. Science. 2007;318(5849):426-30.

14. He W, Dong C, Sefei Y, Dawei Z, Kui X, Xiaogang L. Facile incorporation of hydroxyapatite onto an anodized Ti surface via a mussel inspired polydopamine coating. Appl Surf Sci. 2016;378:496-503.

15. Ma T, Ge X, Zhang Y, et al. Effect of titanium surface modifications of dental implants on rapid osseointegration. In: Suzuki KSO, Takahashi N, editors. Oral Health Science 2016 Innovative Research on Biosis-Abiosis Intelligent Interface. Singapore: Springer; 2016. p. 247-56.

16. Shim JH, Moon TS, Yun MJ, Jeon YC, Jeong CM, Cho DW, et al. Stimulation of healing within a rabbit calvarial defect by a PCL/ PLGA scaffold blended with TCP using solid freeform fabrication technology. J Mater Sci Mater Med. 2012;23:2993-3002

17. Chien CY, Liu TY, Kuo WH, et al. Dopamine-assisted immobilization of hydroxyapatite nanoparticles and RGD peptides to improve the osteoconductivity of titanium. J Biomed Mater Res A. 2013;101(3):740-7.

18. Batul R, Tamanna T, Khaliq A, et al. Recent progress in the biomedical applications of polydopamine nanostructures. Biomater Sci. 2017 Jun 27; 5(7):1204-29.

19. Liu Y, Ai K, Lu L. Polydopamine and its derivative materials: synthesis and promising applications in energy, environmental, and biomedical fields. Chem Rev. 2014;114(9):5057-115.

20. Woehlk H, Lauer A, Trouillet $\mathrm{V}$, et al. Dynamic nitroxide functional materials. Chemistry. 2018;24(71):18873-9.

\section{Publisher's Note}

Springer Nature remains neutral with regard to jurisdictional claims in published maps and institutional affiliations. 